\title{
Downregulation of LRIG2 expression inhibits angiogenesis of glioma via EGFR/VEGF-A pathway
}

\author{
HONG-KUAN YANG, HAO CHEN, FENG MAO, QUN-GEN XIAO, RUI-FAN XIE and TING LEI \\ Department of Neurosurgery, Tongji Hospital, Tongji Medical College, \\ Huazhong University of Science and Technology, Wuhan, Hubei 430030, P.R. China
}

Received February 5, 2017; Accepted June 22, 2017

DOI: $10.3892 / 01.2017 .6671$

\begin{abstract}
Active angiogenesis is the basic pathological feature of glioma. Tumor angiogenesis is involved in vascular endothelial cell migration to the tumor tissue and in the formation of tube-like structures. The present study aimed to investigate the role of leucine-rich repeats and immunoglobulin-like domains 2 (LRIG2) in glioma angiogenesis. Glioma $(n=50)$ and normal brain $(n=20)$ tissue samples were collected from patients to detect the expression of LRIG2, epidermal growth factor receptor (EGFR), vascular endothelial growth factor A (VEGF-A), and cluster of differentiation 31 (CD31) using immunohistochemistry. In addition, the association between the expression of LRIG2 in glioma tissue and the microvessel density (MVD) was analyzed. In vitro, the expression of LRIG2 in human glioma U87 and U251 cell lines was knocked down. Subsequently, cell migration and tube formation assays of human umbilical vein endothelial cells (HUVECs) were performed using a coculture system. The protein expression levels of LRIG2, EGFR, phosphorylated-EGFR and VEGF-A were determined using western blotting. The results demonstrated that the expression levels of LRIG2, EGFR, VEGF-A and CD31 were highly upregulated in glioma tissue samples. Furthermore, LRIG2 expression in glioma tissue samples was significantly correlated with the MVD. In vitro, the downregulation of LRIG2 inhibited HUVEC migration and tube formation induced by coculture with glioma cells. The downregulation of LRIG2 resulted in decreased expression of EGFR and VEGF-A. The effects of the LRIG2 knockdown were reversed following EGF treatment. These findings suggest that LRIG2 is a potential target for the inhibition of glioma angiogenesis, which is possibly mediated via the EGFR/VEGF-A signaling pathway.
\end{abstract}

Correspondence to: Professor Hong-Kuan Yang, Department of Neurosurgery, Tongji Hospital, Tongji Medical College, Huazhong University of Science and Technology, 1095 Jiefang Avenue, Wuhan, Hubei 430030, P.R. China

E-mail: hongkuanyang@yeah.net

Key words: LRIG2, angiogenesis, glioma, EGFR, VEGF-A

\section{Introduction}

Malignant glioma is one of the most common primary central nervous system tumors, which account for more than $70 \%$ of intracranial malignant tumors (1). The character of malignant glioma is diffuse growth and highly invasive (1). Active angiogenesis is the basic pathological feature of most malignant tumors (including glioma), and plays an important role in the occurrence, development and treatment of tumors (2). Tumor angiogenesis is not only associated with vascular endothelial cell division and proliferation. Extracellular matrix remodeling is also associated with vascular endothelium cell migration to the tumor tissue and tube-like structure formation (3). Recently, many studies have launched to understand the mechanism of glioma angiogenesis, to search for more effective treatment targets and therapeutics to extend the asymptomatic survival time and improve the prognosis of glioma patients.

Angiogenesis of glioma is influenced by various factors in the tumor microenvironment, in which vascular endothelial growth factor A (VEGF-A) is considered as the key factor and is highly expressed in glioma (4). The VEGF-A secreted by glioma is an important source of tumor angiogenesis microenvironment. It binds to the VEGF-A receptor (VEGFR), then activates its downstream signaling pathway, stimulates tumor vascular endothelial cell proliferation, migration and tube-like structure formation, finally promotes tumor neovascularization (5). Therefore, inhibition of tumor angiogenesis targeting VEGF-A is considered to be an effective treatment for glioma.

The secretion VEGF-A is regulated by various factors. One of the critical regulatory pathways is the epidermal growth factor receptor (EGFR) signaling pathway (6). The main downstream pathway to regulate VEGF expression of EGFR signaling pathway is PI3K-AKT, RAS-MAPK and JAK-signal transducer and activator of transcription (STAT) signaling pathway (7). EGFR downstream signaling pathway affects transcription factors such as STAT, transcription factor second component 1 (SP1) and hypoxia-inducible factor (HIF) (8). These three transcription factors bind to the promoter of VEGF-A mRNA, then promote the expression of VEGF-A mRNA and increase the secretion of VEGF-A (8). The overexpression, mutation or gene amplification of EGFR is found in most malignant glioma, which abnormal activates EGFR signaling pathway, results in increased secretion level of VEGF-A (8). Hence, studying the mechanism of VEGF 
secretion through EGFR signaling pathway is expected to achieve the purpose of inhibiting angiogenesis of glioma.

Previous studies show that EGFR signaling pathway was regulated by the leucine-rich repeats and immunoglobulin-like domains (LRIG) family which contains LRIG1, 2 and 3 (7). The LRIG family expresses in various normal tissues and tumors (9-12). LRIG1 and 3 which are considered as tumor suppressor genes, inhibit a variety of tyrosine kinase receptors such as EGFR, Met and Ret, thus affect tumor biological characteristics (13-16).

However,LRIG2 is used as a prognostic indicator of cervical cancer and glioma patients, whose survival time is negatively correlated with the cytoplasm staining of LRIG2 $(11,17,18)$. A preliminary study in our laboratory suggests that downregulation of LRIG2 leads to decreasing phosphorylation of EGFR, inhibit cell growth and promote cell apoptosis in a human glioma cell line (GL15) (19). However, the effect of LRIG2 on glioma angiogenesis is not well known.

In the present study, we tested the expression of LRIG2, EGFR, VEGF-A and CD31 in glioma tissues and normal brain tissues, then analyzed the correlation between LRIG2 in glioma tissues and the MVD. In vitro, we studied the possible effects of downregulation of LRIG2 on HUVEC migration and tube formation induced by coculture with glioma cells. These findings clearly demonstrate that LRIG2 is a potential target in glioma angiogenesis, which is possibly mediated via the EGFR/VEGF-A pathway.

\section{Materials and methods}

Regents. Human glioma cell lines U87 and U251 cells, human umbilical vein endothelial cell (HUVEC) was obtained from Cell Bank, Chinese Academy of Sciences, (Shanghai, China). Dulbecco's Modified Eagle's Medium (DMEM) and fetal bovine serum (FBS) were obtained from Thermo Fisher Scientific (Waltham, MA, USA). Anti-LRIG2 antibody (ab157492), anti-EGFR antibody (ab52894), anti-VEGF-A antibody (ab46154), anti-CD31 antibody (ab28364), anti-p-EGFR antibody (ab40815) and biotinylated anti-rabbit immunoglobulin $\mathrm{G}$ were obtained from Abcam (Cambridge, MA, USA). Matrigel was obtained from BD Bioscience (San Diego, CA, USA). HTS Transwell-24 Well Permeable Supports were obtained from Corning Life Sciences (Corning, CA, USA). TRIzol reagent, reverse transcription kit and SYBR-Green qPCR SuperMix were obtained from Invitrogen Life Technologies (Carlsbad, CA, USA). BCA protein assay kit was obtained from Beyotime Institute of Biotechnology (Nantong, China). PVDF membranes were obtained from Pall Corporation (New York, NY, USA). HRP substrate was obtained from Millipore (Billerica, MA, USA). Streptavidin-biotinylated horseradish peroxidase complex was obtained from Santa Cruz Biotechnology, Inc. (Dallas, TX, USA). DAB was obtained from DakoCytomation (Carpinteria, CA, USA). Cresyl violet counterstaining was obtained from Sigma-Aldrich (St. Louis, MO, USA).

Ethical statement. This study was performed with the approval of research ethic committee of Huazhong University of Science and Technology. All subjects provided written informed consent.
All the procedures involving subjects were performed in the study after obtaining ethical approval of the Medical Ethics Committee of Huazhong University of Science and Technology.

Tissue samples. Glioma tissues $(\mathrm{n}=50)$ and normal brain tissues $(n=20)$ were collected from Department of Neurosurgery, Tongji Hospital, Tongji Medical College, Huazhong University of Science and Technology, from 2012 to 2015, as study samples with complete clinicopathological data.

Immunohistochemistry. The expression of LRIG2, EGFR, VEGF-A and CD31 in the normal brain tissues and glioma tissues was detected using immunohistochemistry. The tissues were transferred to $4 \%$ paraformaldehyde, and embedded in paraffin. The tissue was cut into $3-\mu \mathrm{m}$ serial sections using a Leica microtome (Leica Microsystems GmbH, Wetzlar, Germany). IHC was performed according to a previously published protocol. Briefly, sections were incubated in $3 \% \mathrm{H}_{2} \mathrm{O}_{2}$ in PBS for $7 \mathrm{~min}$, washed with PBS three times, treated with BSA $0.3 \%$ Triton X-100 for 2 h. Further, the sections were incubated overnight at $4^{\circ} \mathrm{C}$ with rabbit anti-LRIG2 antibodies (1:750), rabbit anti-EGFR antibodies (1:500), rabbit anti-VEGF-A antibodies (1:500) or rabbit anti-CD31 antibodies (1:500). After washing twice with PBS, sections were treated for $40 \mathrm{~min}$ with biotinylated anti-rabbit immunoglobulin $\mathrm{G}$, washed three times, and processed using streptavidin-biotinylated horseradish peroxidase complex. The reaction was visualized using DAB and cresyl violet counterstaining. Images were obtained using a microscope (Olympus Corporation, Tokyo, Japan).

Evaluation of immunohistochemical staining. The scoring standards were defined as follows (20). Briefly, the immunoreactive score (IRS) was derived by multiplying the positive cell staining intensity (SI) with the percentage of positive cells (PP). SI was judged from 0 to 3 points: 0 , no staining; 1 , weakly positive staining; 2 , moderately positive staining; and 3 , strongly positive staining; PP was judged from 0 to 4 points: 0, no staining; $1,1-10 \% ; 2,11-50 \% ; 3,51-80 \%$; and 4, 80-100\%.

Assessment of microvessel density (MVD). The vessels of tissues were assessed by immunohistochemical staining for CD31 in three areas of highest vascular density per section of tumor specimen (x400 magnification) as described before (21). Vessels were counted in 10 high power fields the average was considered as MVD. All clinical data were blinded to the pathologist.

Culture and transfection of the cell lines. Human glioma cell lines U87 and U251 were cultured in DMEM medium supplemented with $10 \% \mathrm{FBS}$ and penicillin/streptomycin under $5 \% \mathrm{CO}_{2}$ in air at $37^{\circ} \mathrm{C}$. The cells were seeded in 24-well plates and cultured for $24 \mathrm{~h}$. Subsequently, cells were transfected with LRIG2 siRNA (si-LRIG2) or negative control siRNA (si-NC) with Lipofectamine ${ }^{\circledR} 2000$ reagent according to the manufacturer's instruction. The sequences for si-LRIG2 and si-NC are as follows: si-LRIG2-sense: 5'-UCGGUUGUCUAA CUGGAACTT-3', si-LRIG2-antisense: 5'-GUUCCAGUU AGACAACCGATT-3', si-NC-sense: 5'-UUCUCCGAACGU 
GUCACGUTT-3', and si-NC-antisense: 5'-ACGUGACACG UUCGGAGAATT-3'.

Tube formation assay under co-culture system. To investigate tube formation ability of HUVEC induced by coculture with glioma cells, the tube formation assay was detected by using co-culture system $(0.4 \mu \mathrm{m}$ pore size $)$ as described before. Briefly, $50 \mu \mathrm{l}$ diluted matrigel was prepared and added to the upper chamber of each transwell chamber, then incubated at $37^{\circ} \mathrm{C}$ for $30 \mathrm{~min}, \mathrm{UV}$ irradiation overnight, jelling for $30 \mathrm{~min}$ before the formal experiment. U87 and U251 cells ( $1 \times 10^{5} /$ well) were seeded on the lower chamber for $24 \mathrm{~h}$, then cocultivated with HUVECs (1×10\%/well) in the upper chamber. After $24 \mathrm{~h}$, the number of tubular structures was counted and photographed using an inverted microscope. All the experiments were repeated in triplicate.

Cell migration assay under co-culture system. To investigate the migration ability of HUVEC induced by coculture with glioma cells, the cell migration assay was detected by using co-culture system ( $8 \mu \mathrm{m}$ pore size) as described before. Briefly, U87 and U251 cells (1x10\% $/$ well) were seeded on the lower chamber for $24 \mathrm{~h}$, then cocultivation with HUVECs $\left(1 \times 10^{5} /\right.$ well $)$ in the upper chamber. After $24 \mathrm{~h}$, HUVECs on the upper surface of the membrane were then removed by cotton swabs. The migrated cells were fixed with $10 \%$ methanol and stained with $0.1 \%$ crystal violet. Then the migrated cells were counted under a microscope. All the experiments were repeated in triplicate.

Quantitative PCR ( $q P C R$ ). U87 and U251 cells were seeded in 6-well plates and received the indicated treatment. After treatment for $24 \mathrm{~h}$, total RNA was extracted using Trizol reagent according to the manufacturer's instructions. The primer sequences for LRIG2 and $\beta$-actin are as follows: LRIG2, forward: 5'-CATGTGCCCTCACTACCA-3', and reverse: 5'-CTCCAGGACCCGAGAATA-3'; $\beta$-actin, forward: 5'-TGG CACACCTTCTACAA-3', and reverse: 5'-AGCCTGGAT AGCAACGTACA-3'. Reverse transcription and qPCR were performed in accordance with the protocol recommended by the manufacturers of SYBR-Green qPCR SuperMix. The relative expression of mRNA was assessed by the comparative $2^{-\Delta \Delta \mathrm{Cq}}$ method. $\beta$-actin was used as an internal standard.

Western blotting. Each sample was lysed using RIPA buffer. Total protein concentration was determined with a BCA protein assay kit, according to the manufacturer's instruction. Equal amounts of total protein were separated in $10 \%$ SDS-polyacrylamide gels and transferred to PVDF membranes. After blocking with 5\% milk in TBS containing $0.05 \%$ Tween-20 (TBST) for $1 \mathrm{~h}$ at $37^{\circ} \mathrm{C}$, membranes were incubated for 40 min with anti-LRIG2 antibody $(1: 1,000)$, anti-EGFR antibody $(1: 1,000)$, anti-VEGF-A antibody (1:1,000), anti-p-EGFR antibody (1:750), washed with TBST and incubated with secondary antibody, and visualized using immobilon western chemiluminescent HRP substrate.

Statistical analysis. SPSS 19.0 (SPSS, Inc., Chicago, IL, USA) was employed for statistical analysis. All data were expressed as the mean \pm standard deviation. Differences between two groups were assessed using Student's t-test. The differences between multiple groups were assessed using one-way ANOVA or Kruskal-Wallis H tests. Correlation analysis of two variables was assessed using Pearson correlation coefficient. $\mathrm{P}<0.05$ was considered to indicate a statistically significant difference.

\section{Results}

LRIG2 was upregulated in glioma tissues and was directly correlated with MVD. We used immunochemistry assay to analyze the protein expression (evaluated by IRS) of LRIG2, EGFR, VEGF-A and CD31 in glioma tissues and normal brain tissues. As shown in Table I, with the increase of tumor malignancy, the expression of LRIG2, EGFR, VEGF-A and CD31 was significantly enhanced. The expression of LRIG2, EGFR, VEGF-A and CD31 was significantly higher in malignant gliomas than that in low grade. As shown in Fig. 1A, the expression level of these four protein was highly expressed in glioma tissues. However, the level of these four protein was not expressed or low expressed in normal tissues (Fig. 1B).

Then the correlation between the expression of LRIG2, EGFR, VEGF-A in 50 glioma tissues and the MVD were further analyzed. The results showed that LRIG2, EGFR, VEGF-A expression in glioma tissues remained significantly correlated to the MVD, respectively (Fig. 1C).

The effects of RNA Interference. U87 and U251 cells were transfected with si-LRIG2 or si-NC, then the mRNA level and the protein level of LRIG2 were determined. As shown in Fig. 2A, when compared with the NC group, the LRIG2 mRNA expression was significantly decreased when transfected with si-LRIG2 both in U87 and U251 cell lines. Consistent with the results of qPCR detection, the protein level of LRIG2 analyzing by western blotting was significantly decreased in si-LRIG2 transfected group, when compared with the NC group (Fig. 2B).

Downregulation of LRIG2 in glioma cell lines inhibited tube formation and cell migration of HUVECs. To observe the effect of downregulation of LRIG2 on HUVECs angiogenesis. We knockdown the expression of LRIG2 in glioma cells, then tube formation was assessed by using transwell co-cultured model. The number of formed tubular structures were was significantly decreased when transfected with si-LRIG2 both in U87 and U251 cell lines, when compared with the NC group (Fig. 3A and C).

Then we analyzed the effect of downregulation of LRIG2 on HUVECs migration. As shown in Fig. 3B and D, when compared with the $\mathrm{NC}$ group, the migrated cells were was significantly decreased when transfected with si-LRIG2 both in U87 and U251 cells.

Downregulation of LRIG2 decreased the expression of EGFR and VEGF-A. The protein expression level of EGFR, p-EGFR and VEGF-A was assessed by Western blot assay. The results showed that EGFR, p-EGFR and VEGF-A expression was significantly decreased when transfected with si-LRIG2 both in U87 and U251 cells, when compared with the NC group (Fig. 4). 
Tabel I. Expression of LRIG2, EGFR, VEGF-A and CD31 in normal brains and gliomas.

\begin{tabular}{lccccc}
\hline Group & No. & LRIG2 protein (IRS) & EGFR protein (IRS) & VEGF-A protein (IRS) & CD31 protein (MVD) \\
\hline Normal brain & 20 & $0.90 \pm 0.64$ & $0.15 \pm 0.37$ & $0.55 \pm 0.51$ & $5.35 \pm 2.62$ \\
Grade I-II & 24 & $3.25 \pm 2.80$ & $3.46 \pm 3.44$ & $4.08 \pm 3.65$ & $13.25 \pm 5.00$ \\
Grade III-IV & 26 & $8.12 \pm 3.15^{\mathrm{a}}$ & $6.96 \pm 3.82^{\mathrm{a}}$ & $6.12 \pm 4.20^{\mathrm{a}}$ & $31.88 \pm 5.05^{\mathrm{a}}$ \\
\hline
\end{tabular}

LRIG2, leucine-rich repeats and immunoglobulin-like domains 2; EGFR, epidermal growth factor receptor; VEGF-A, vascular endothelial growth factor A; CD31, cluster of differentiation 31; IRS, immunoreactive score; MVD, microvessel density. ${ }^{\text {a }}<0.01$ vs. the Grade I-II group.

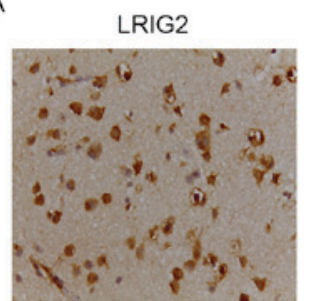

B

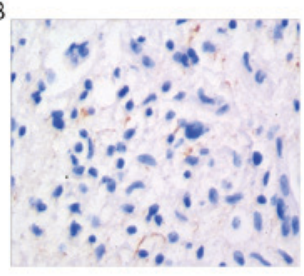

EGFR
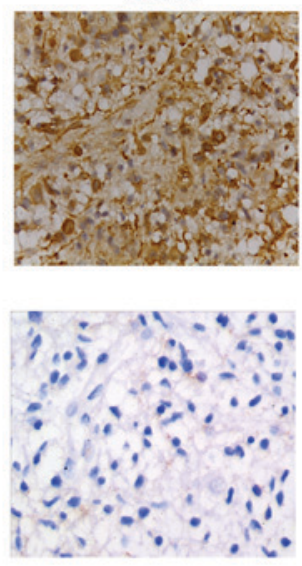

VEGF-A
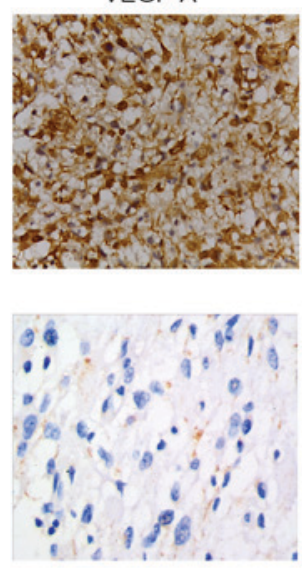

CD31
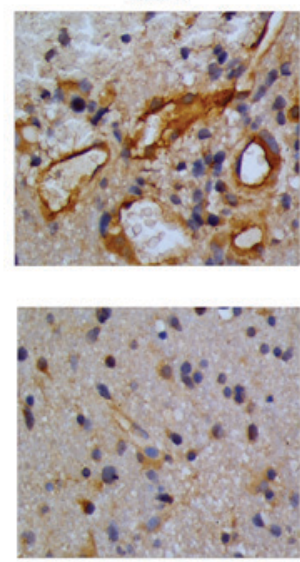

C
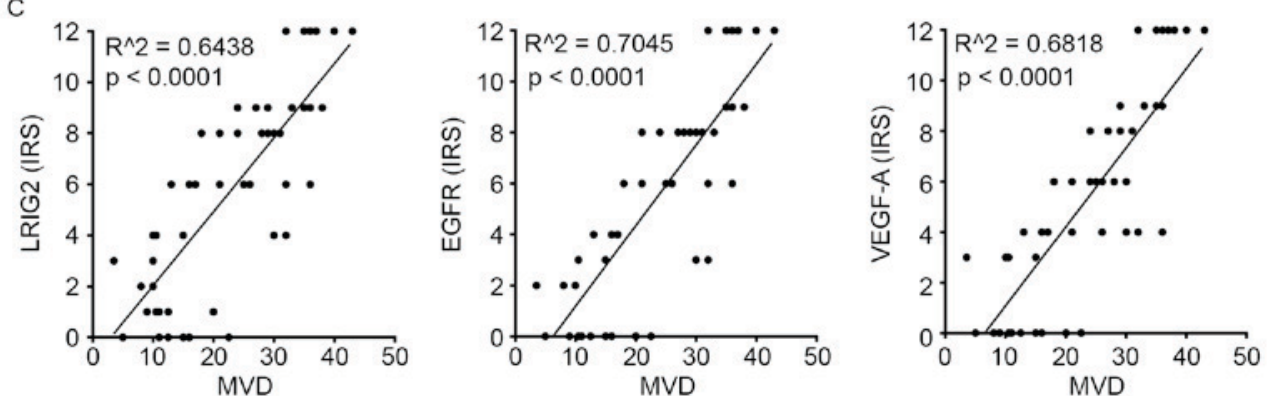

Figure 1. We used immunochemistry assay to analyze the protein expression (evaluated by immunoreactive score) of LRIG2, EGFR, VEGF-A and CD31 in glioma tissues and normal brain tissues. The vessels of tissues were expressed by immunohistochemical staining for CD31 highlight for calculation of MVD. The expression level of LRIG2, EGFR, VEGF-A and CD31 was highly expressed in glioma tissues (A), but was not expressed or lowly expressed in normal tissues (B). The expression of LRIG2, EGFR, VEGF-A was directly correlated with MVD. (C) Results are representative of three different experiments. Original magnification, x400. LRIG2, leucine-rich repeats and immunoglobulin-like domains 2; EGFR, epidermal growth factor receptor; VEGF-A, vascular endothelial growth factor A; CD31, cluster of differentiation 31; MVD, microvessel density.

A

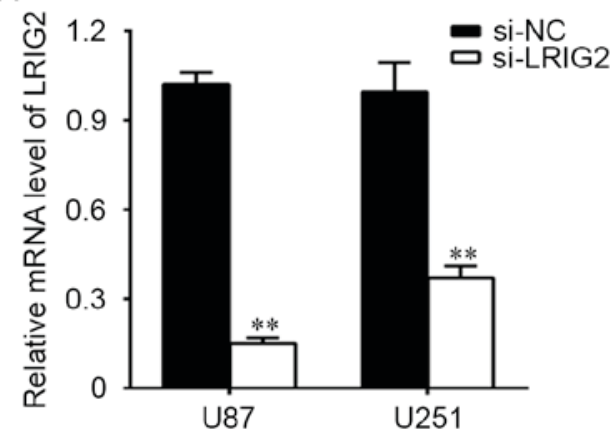

B

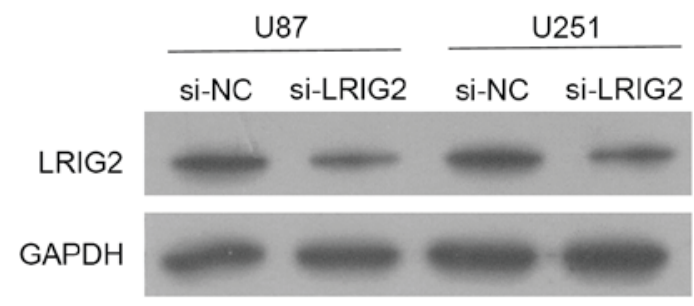

Figure 2. The U87 and U251 cells were transfected with si-LRIG2 or si-NC. (A) The mRNA level of LRIG2 was determined by qPCR. (B) The protein level of LRIG2 was determined by western blotting. ${ }^{* *} \mathrm{P}<0.01$ vs. the si-NC group. Results are representative of three different experiments. si-LRIG2, LRIG2 siRNA; si-NC, negative control siRNA. 
A

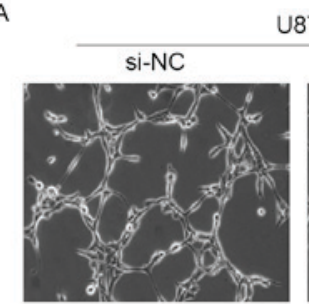

บ87

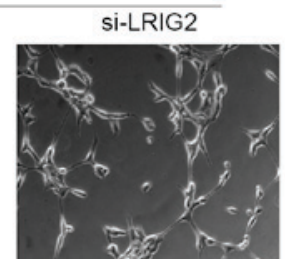

B

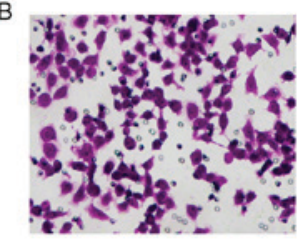

C

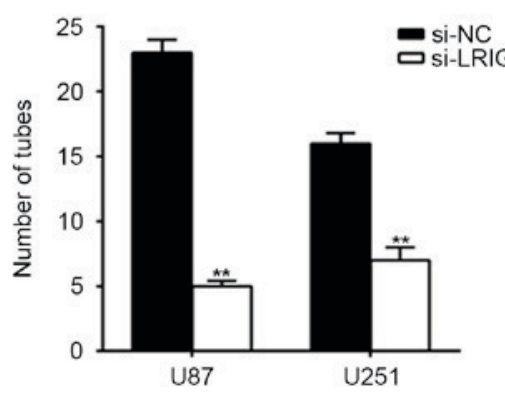

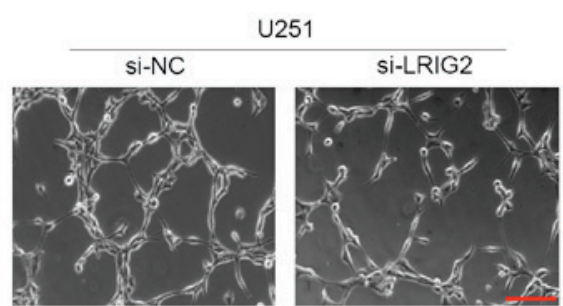

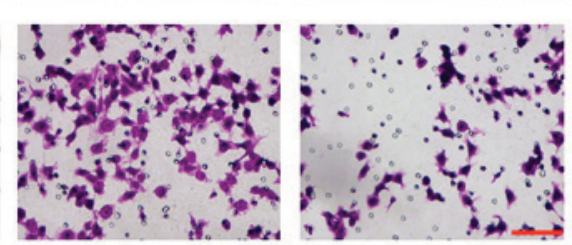

D

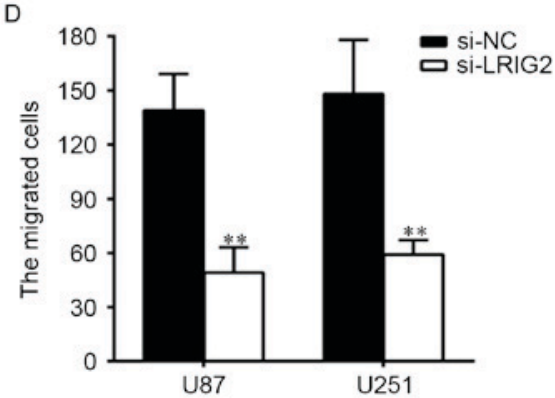

Figure 3. Downregulation of LRIG2 in glioma cell lines inhibited tube formation and cell migration of HUVECs. U87 and U251 cell lines were transfected with si-LRIG2 or si-NC. (A) HUVECs angiogenesis induced by coculture with glioma cells was assessed by using transwell co-cultured system ( $0.4 \mu \mathrm{m}$ pore size). (B) HUVECs migration induced by coculture with glioma cells was assessed by using transwell co-cultured system (8 $\mu$ m pore size). (C) The number of formed tubular structures were calculated. (D) The migrated cells were counted. ${ }^{* *} \mathrm{P}<0.01$ vs. the si-NC group. Results are representative of three different experiments. The scale bar is $100 \mu \mathrm{m}$. si-LRIG2, LRIG2 siRNA; si-NC, negative control siRNA; HUVECs, human umbilical vein endothelial cells.

Downregulation of LRIG2 in glioma cell lines inhibited tube formation and cell migration of HUVECs via inhibition of EGFR/VEGF-A pathway. EGFR could be activated by EGF, hence we added EGF into the glioma cells to observe the potential involvement of EGFR/VEGF-A pathway. U87 and U251 cell lines were transfected with si-LRIG2 or NC siRNA, then treated with $100 \mathrm{ng} / \mathrm{ml}$ EGF or $\mathrm{DDH}_{2} \mathrm{O}$. The results showed that EGF treatment in si-NC transfected glioma cells significantly enhanced the number of formed tubular structures (Fig. 5A and C) and cell migration (Fig. 5B and D) in HUVECs, when compared to the $\mathrm{DDH}_{2} \mathrm{O}$ treatment group. The expression of EGFR, p-EGFR and VEGF-A was further increased (Fig. 6). However, the enhanced effect of EGF was diminished in si-LRIG2-transfected glioma cells (Figs. 5 and 6).

\section{Discussion}

Tumor angiogenesis is not only associated with vascular endothelial cell division proliferation, extracellular matrix remodeling, but is also related to vascular endothelium cell migration to the tumor tissue and tube-like structure formation. In this study, we revealed the role of LRIG2 in glioma angiogenesis. The results showed that the expression level of LRIG2, EGFR, VEGF-A and CD31 was highly expressed in glioma tissues. The LRIG2 expression in glioma tissues was positively correlation with the MVD. Downregulation of LRIG2 inhibited HUVEC migration and tube formation induced by coculture with glioma cells. Mechanistically, downregulation of LRIG2 decreased the expression of EGFR,
A

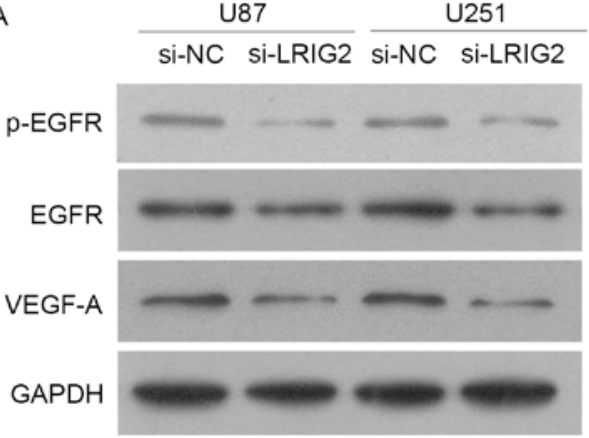

B

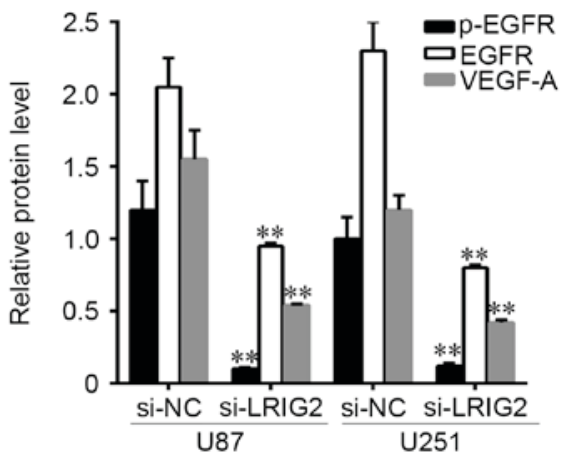

Figure 4. Downregulation of LRIG2 decreased the expression of EGFR and VEGF-A. (A) The protein expression level of EGFR, p-EGFR and VEGF-A were assessed by western blot assay. (B) The densitometry data of western blotting. ${ }^{* *} \mathrm{P}<0.01$ vs. the si-NC group. Results are representative of three different experiments. LRIG2, leucine-rich repeats and immunoglobulin-like domains 2; EGFR, epidermal growth factor receptor; VEGF-A, vascular endothelial growth factor A; si-LRIG2, LRIG2 siRNA; si-NC, negative control siRNA. 
A
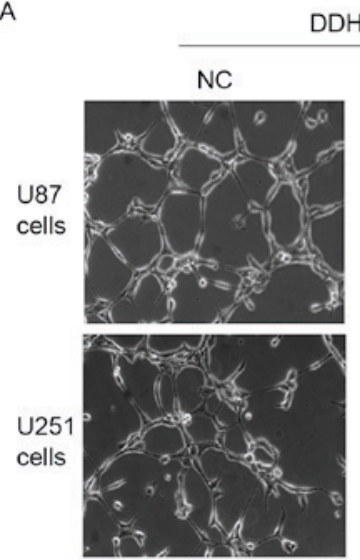

$\mathrm{DH}_{2} \mathrm{O}$

Si-LRIG2
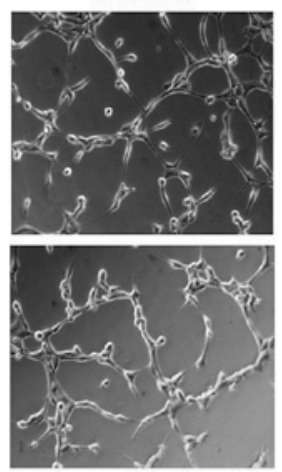

B
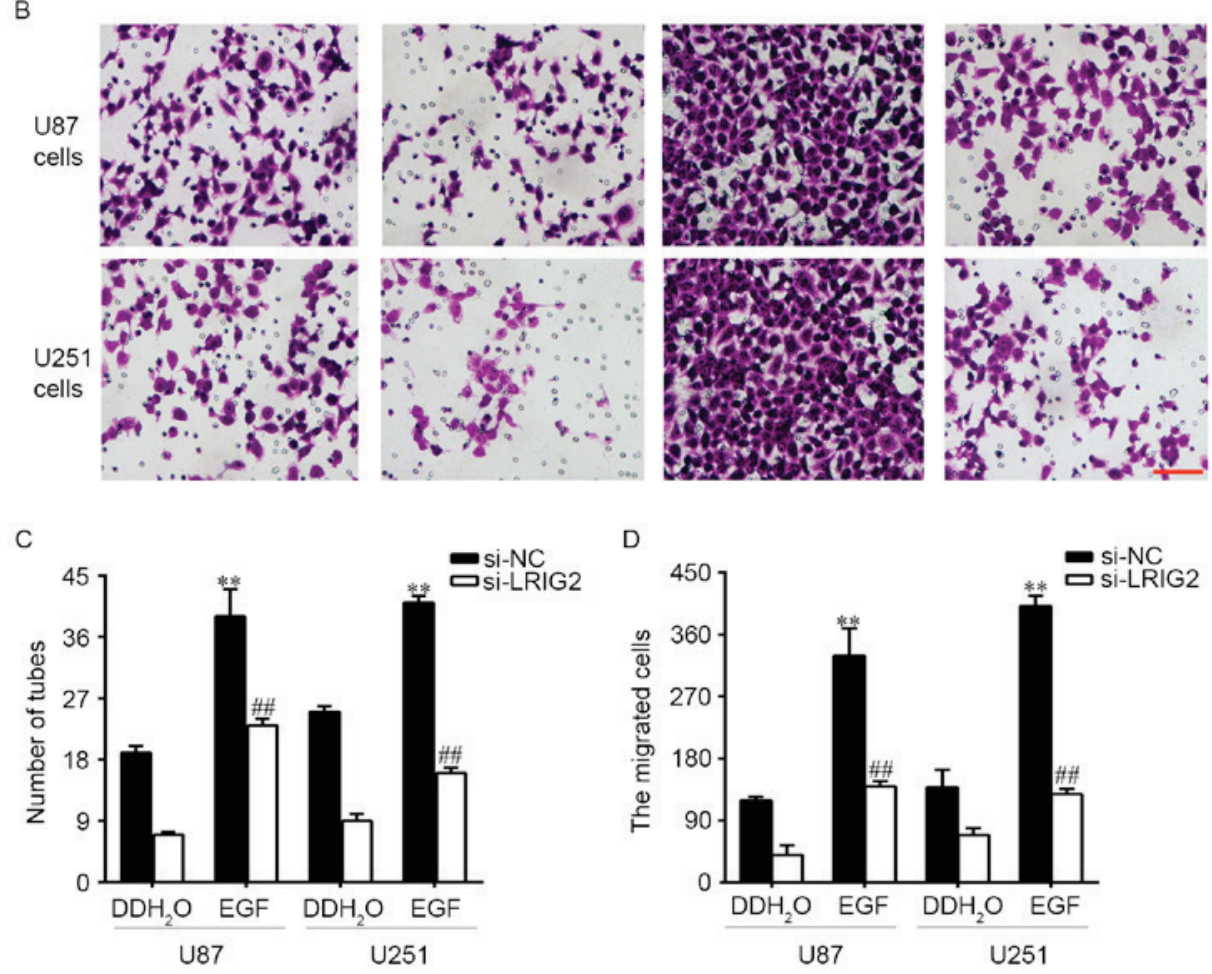

Figure 5. U87 and U251 cell lines were transfected with si-LRIG2 or si-NC, then treated with $100 \mathrm{ng} / \mathrm{ml} \mathrm{EGF} \mathrm{or} \mathrm{DDH}_{2} \mathrm{O}$ for $24 \mathrm{~h}$. (A) HUVECs angiogenesis was assessed by using transwell co-cultured system $(0.4 \mu \mathrm{m}$ pore size). (B) HUVECs migration was assessed by using transwell co-cultured system $(8 \mu \mathrm{m}$ pore size). Then the number of $(\mathrm{C})$ formed tubular structures and $(\mathrm{D})$ the migrated cells was calculated. ${ }^{* *} \mathrm{P}<0.01 \mathrm{vs}$. the si-NC $+\mathrm{DDH}{ }_{2} \mathrm{O}$ group. ${ }^{\# \#} \mathrm{P}<0.01$ vs. the si-LRIG2 $+\mathrm{DDH}_{2} \mathrm{O}$ group. Results are representative of three different experiments. The scale bar is $100 \mu \mathrm{m}$. LRIG2, leucine-rich repeats and immunoglobulin-like domains 2; EGF, epidermal growth factor; HUVECs, human umbilical vein endothelial cells; si-LRIG2, LRIG2 siRNA; si-NC, negative control siRNA.

p-EGFR and VEGF-A. The decreased effect was diminished by EGF treatment.

LRIG2 is one of the members of the LRIG gene family, which include three members: LRIG1 (gene no. AF381545), LRIG2 (gene no. AY505340), LRIG3 (gene no. AY505341). The gene products of LRIG gene family have a unique protein structure: a signal peptide, 15 consecutive leucine-rich repeats (LRRs), three immunoglobulin-like regions (Ig-like), a transmembrane segment and an intracellular fraction. Previous studies demonstrate that LRIG1 negatively regulates tumor growth and development and is viewed as a tumor suppressor (15). However, LRIG2 might have a function distinct from that of LRIG1, and possibly contributing to the etiology of oligodendroglioma (18). LRIG2 acts as a prognostic indicator of various cancers such as cervical cancer, (12) non-small cell lung cancer (22) and glioma (18). Consistent with the previous study, our study found that the expression level of LRIG2 was highly expressed in glioma tissues, but was not expressed or low expressed in normal brain tissues.

Research confirmed that glioma is a highly vascularize human tumor, and its proliferation and invasion are dependent on tumor angiogenesis (2). CD31 is a vascular endothelial cell marker, which is used primarily to study tumor angiogenesis. MVD is an important index for the detection of tumor angiogenesis which is assessed by the immunochemistry staining of CD31. Consistent with Cui et al (23), our study revealed that the expression of CD31 was highly expressed in glioma tissues, which indicated that angiogenesis in glioma was higher than 
A

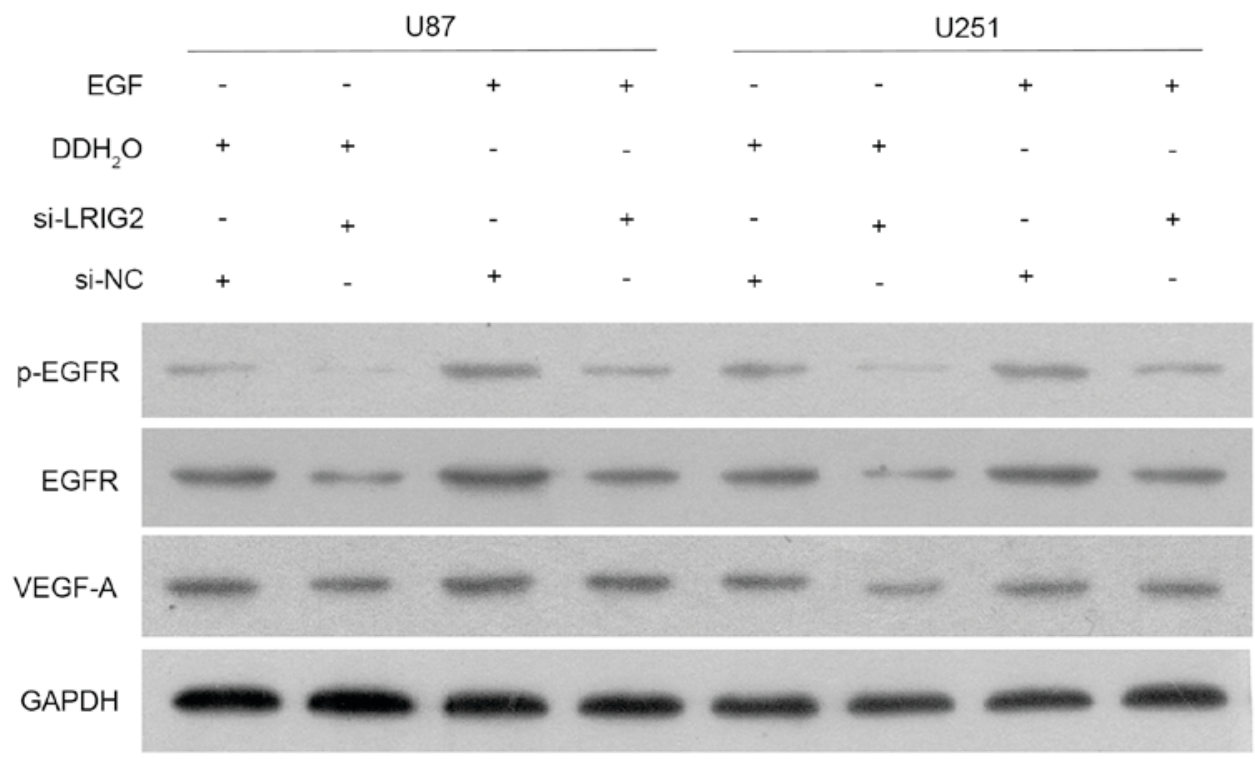

B
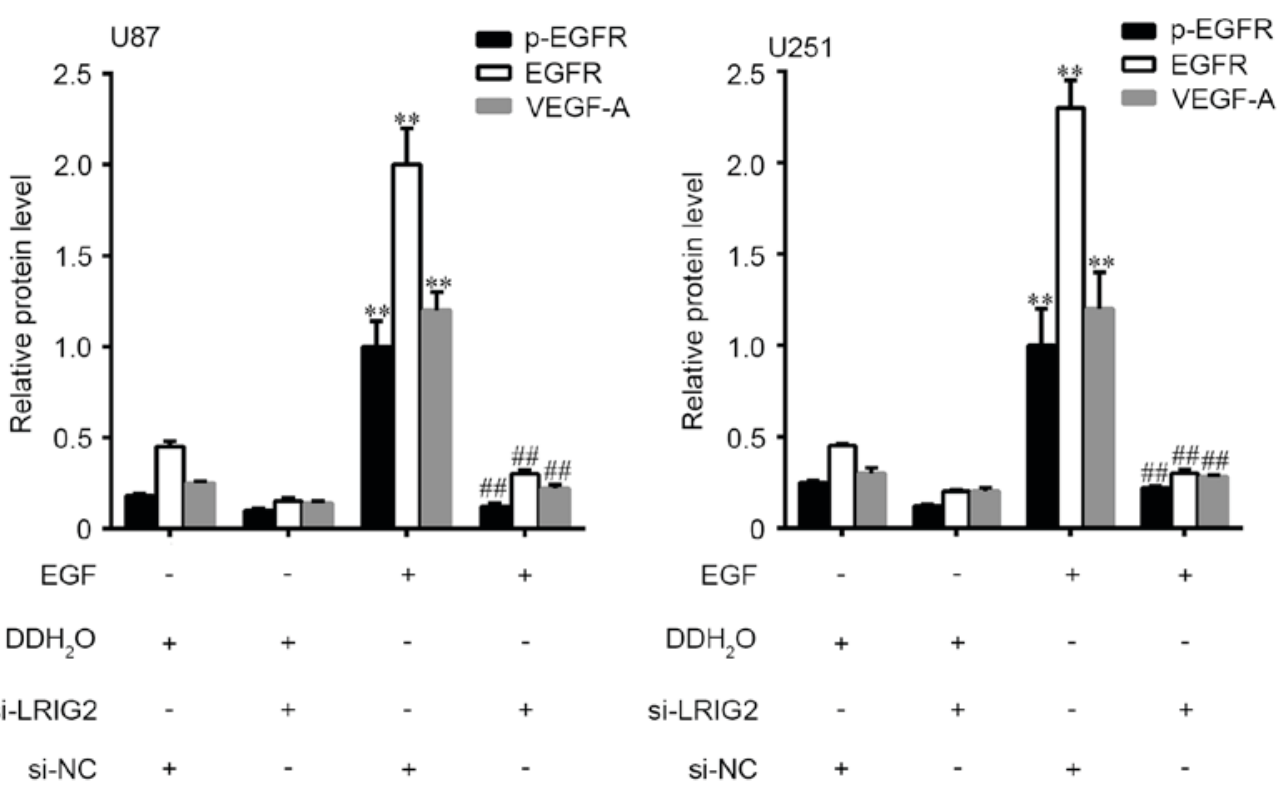

Figure 6. U87 and U251 cell lines were transfected with si-LRIG2 or si-NC, then treated with $100 \mathrm{ng} / \mathrm{ml} \mathrm{EGF}$ or $\mathrm{DDH}_{2} \mathrm{O}$ for $24 \mathrm{~h}$. (A) The protein expression level of EGFR, p-EGFR and VEGF-A were assessed by western blot assay. (B) The densitometry data of western blotting. ${ }^{* *} \mathrm{P}<0.01$ vs. the si-NC $+\mathrm{DDH} 2 \mathrm{O}$ group. ${ }^{\# /} \mathrm{P}<0.01$ vs. the si-LRIG2 + $\mathrm{DDH}_{2} \mathrm{O}$ group. Results are representative of three different experiments. LRIG2, leucine-rich repeats and immunoglobulin-like domains 2; EGFR, epidermal growth factor receptor; VEGF-A, vascular endothelial growth factor A; si-LRIG2, LRIG2 siRNA; si-NC, negative control siRNA.

in normal brain tissues. LRIG2 expression in glioma tissues was positivity correlated with the MVD. It is suggested that the expression of LRIG2 in human glioma might be related to tumor angiogenesis, which might be related to the regulation of LRIG2.

Our previous study demonstrated that knockdown of LRIG2 by RNA interference inhibited glioma cell (GL15) growth, caused cell cycle redistribution and increased cell apoptosis in vitro, suggested that LRIG2 was an attractive target in glioma therapy (19). In this study, we further revealed that downregulation of LRIG2 inhibited the number of formed tubular structures and cell migration of HUVECs induced by glioma cells, indicated that knockdown of LRIG2 inhibited glioma angiogenesis. However, the results showed that the presence of ectodomain of LRIG2 in the culture medium of si-LRIG2 treated cells. Studies using conditioned culture medium by si-LRIG2 treated cell still need studying in the future.

EGFR promotes the expression and secretion of VEGF-A through its downstream transcription factors including STAT, SP1 and HIF. (8) VEGF-A binds to VEGFR, then stimulates vascular endothelial cell proliferation, migration and tube-like structure formation, finally promotes tumor neovascularization (8). Previous study reported that anti-EGFR and VEGF/VEGFR therapy significantly prolonged survival of patients with cancers $(24,25)$. Therefore, inhibition of tumor angiogenesis targeting EGFR/VEGF-A is considered to be an effective treatment for glioma. A preliminary study in our laboratory suggested that downregulation of LRIG2 decreased phosphorylation of EGFR, then result in inhibition of glioma 
cell proliferation. Our study revealed that downregulation of LRIG2 decreased the expression of EGFR, p-EGFR and VEGF-A, then result in anti-angiogenesis of glioma cells. The decreased effect was diminished by EGF (EGFR agonist) treatment. In conclusion, these findings clearly demonstrate that downregulation of LRIG2 is a potential target to inhibit glioma angiogenesis, which is possibly involved in the EGFR/VEGF-A pathway.

\section{Acknowledgements}

This study was supported by the Nature Science Foundation of Hubei Province (no. 2014CFB151).

\section{References}

1. Wen PY and Reardon DA: Neuro-oncology in 2015: Progress in glioma diagnosis, classification and treatment. Nat Rev Neurol 12: 69-70, 2016.

2. Malla RR, Gopinath S, Gondi CS, Alapati K, Dinh DH, Gujrati M and Rao JS: Cathepsin B and UPAR knockdown inhibits tumor-induced angiogenesis by modulating VEGF expression in glioma. Cancer Gene Ther 18: 419-434, 2011.

3. Babykutty S, S PP, J NR, Kumar MA, Nair MS, Srinivas P and Gopala S: Nimbolide retards tumor cell migration, invasion, and angiogenesis by downregulating MMP-2/9 expression via inhibiting ERK1/2 and reducing DNA-binding activity of NF- $\kappa \mathrm{B}$ in colon cancer cells. Mol Carcinog 51: 475-490, 2012.

4. Petrillo M, Borriello M, Fuoco G, Legge F, Iannone V and Ferrandina G: Novel VEGF-independent strategies targeting tumor vasculature: Clinical aspects. Curr Pharm Des 18: 2702-2712, 2012.

5. Reardon DA, Turner S, Peters KB, Desjardins A, Gururangan S, Sampson JH, McLendon RE, Herndon JE II, Jones LW, Kirkpatrick JP, et al: A review of VEGF/VEGFR-targeted therapeutics for recurrent glioblastoma. J Natl Compr Canc Netw 9: 414-427, 2011.

6. Cea V, Sala C and Verpelli C: Antiangiogenic therapy for glioma. J Signal Transduct 2012: 483040, 2012.

7. Casaletto JB and McClatchey AI: Spatial regulation of receptor tyrosine kinases in development and cancer. Nat Rev Cancer 12: 387-400, 2012.

8. Larsen AK, Ouaret D, El Ouadrani K and Petitprez A: Targeting EGFR and $\operatorname{VEGF}(\mathrm{R})$ pathway cross-talk in tumor survival and angiogenesis. Pharmacol Ther 131: 80-90, 2011.

9. Malik U and Javed A: LRIGs: A prognostically significant family with emerging therapeutic competence against cancers. Curr Cancer Drug Targets 17: 3-16, 2017.

10. van Erp S, van den Heuvel DM, Fujita Y, Robinson RA, Hellemons AJ, Adolfs Y, Van Battum EY, Blokhuis AM, Kuijpers M, Demmers JA, et al: Lrig2 negatively regulates ectodomain shedding of Axon guidance receptors by ADAM proteases. Dev Cell 35: 537-552, 2015.

11. Guo D, Nilsson J, Haapasalo H, Raheem O, Bergenheim T, Hedman $\mathrm{H}$ and Henriksson R: Perinuclear leucine-rich repeats and immunoglobulin-like domain proteins (LRIG1-3) as prognostic indicators in astrocytic tumors. Acta Neuropathol 111: 238-246, 2006.
12. Lindström AK, Asplund A and Hellberg D: Correlation between LRIG1 and LRIG2 expressions and expression of 11 tumor markers, with special reference to tumor suppressors, in CIN and normal cervical epithelium. Gynecol Oncol 122: 372-376, 2011.

13. Powell AE, Wang Y, Li Y, Poulin EJ, Means AL, Washington MK, Higginbotham JN, Juchheim A, Prasad N, Levy SE, et al: The pan-ErbB negative regulator Lrig1 is an intestinal stem cell marker that functions as a tumor suppressor. Cell 149: 146-158, 2012.

14. Wong VW, Stange DE, Page ME, Buczacki S, Wabik A, Itami S, van de Wetering M, Poulsom R, Wright NA, Trotter MW, et al: Lrig1 controls intestinal stem-cell homeostasis by negative regulation of ErbB signalling. Nat Cell Biol 14: 401-408, 2012.

15. Lu L, Teixeira VH, Yuan Z, Graham TA, Endesfelder D, Kolluri K, Al-Juffali N, Hamilton N, Nicholson AG, Falzon M, et al: LRIG1 regulates cadherin-dependent contact inhibition directing epithelial homeostasis and pre-invasive squamous cell carcinoma development. J Pathol 229: 608-620, 2013.

16. Bai L, McEachern D, Yang CY, Lu J, Sun H and Wang S: LRIG1 modulates cancer cell sensitivity to Smac mimetics by regulating $\mathrm{TNF} \alpha$ expression and receptor tyrosine kinase signaling. Cancer Res 72: 1229-1238, 2012.

17. Hedman H, Lindström AK, Tot T, Stendahl U, Henriksson R and Hellberg D: LRIG2 in contrast to LRIG1 predicts poor survival in early-stage squamous cell carcinoma of the uterine cervix. Acta Oncol 49: 812-815, 2010.

18. Holmlund C, Haapasalo H, Yi W, Raheem O, Brännström T, Bragge H, Henriksson R and Hedman H: Cytoplasmic LRIG2 expression is associated with poor oligodendroglioma patient survival. Neuropathology 29: 242-247, 2009.

19. Wang B, Han L, Chen R, Cai M, Han F, Lei T and Guo D: Downregulation of LRIG2 expression by RNA interference inhibits glioblastoma cell (GL15) growth, causes cell cycle redistribution, increases cell apoptosis and enhances cell adhesion and invasion in vitro. Cancer Biol Ther 8: 1018-1023, 2009.

20. Remmele W and Stegner HE: Recommendation for uniform definition of an immunoreactive score (IRS) for immunohistochemical estrogen receptor detection (ER-ICA) in breast cancer tissue. Pathologe 8: 138-140, 1987 (In German).

21. Tai YT, Acharya C, An G, Moschetta M, Zhong MY, Feng X, Cea M, Cagnetta A, Wen K, van Eenennaam H, et al: APRIL and BCMA promote human multiple myeloma growth and immunosuppression in the bone marrow microenvironment. Blood 127: 3225-3236, 2016

22. Wang G, Wu J and Song H: LRIG2 expression and prognosis in non-small cell lung cancer. Oncol Lett 8: 667-672, 2014.

23. Cui L, Xu S, Song Z, Zhao G, Liu X and Song Y: Pituitary tumor transforming gene: A novel therapeutic target for glioma treatment. Acta Biochim Biophys Sin (Shanghai) 47: 414-421, 2015.

24. Jiang T, Qiao M, Zhou F, Ren S, Su C and Zhou C: Effect of combined therapy inhibiting EGFR and VEGFR pathways in non-small-cell lung cancer on progression-free and overall survival. Clin Lung Cancer: Dec 29, 2016 (Epub ahead of print).

25. Yu X, Li W, Deng Q, You S, Liu H, Peng S, Liu X, Lu J, Luo X, Yang L, et al: Neoalbaconol inhibits angiogenesis and tumor growth by suppressing EGFR-mediated VEGF production. Mol Carcinog 56: 1414-1426, 2017. 